\title{
Crosstalk between phosphorylation and ubiquitination is involved in high salt-induced WNK4 expression
}

\author{
XIAOYUE ZHAO* , GUANGRUI LAI* , JIANQIAO TU, SHUCHANG LIU and YANYAN ZHAO \\ Department of Clinical Genetics, Shengjing Hospital of China Medical University, Shenyang, Liaoning 110003, P.R. China
}

Received July 24, 2020; Accepted November 24, 2020

DOI: $10.3892 /$ etm.2020.9565

\begin{abstract}
With no lysine 4 (WNK4) is a serine/threonine kinase, which is expressed in the kidney and associated with salt-sensitive hypertension. However, how salt regulates WNK4 remains unclear. In the present study, the C57BL/6 mice and HEK293 cells were treated with high salt and the expression of WNK4 protein and its ubiquitination and phosphorylation levels were detected. Western blotting demonstrated that WNK4 expression was significantly increased in high salttreated mice and cells. Meanwhile, co-immunoprecipitation analysis demonstrated that the ubiquitination of WNK4 was decreased under high-salt simulation. It was also identified that the Lys-1023 site was the most important ubiquitination site for WNK4, and it was found that phosphorylation at the Ser-1022 site was a prerequisite for ubiquitination. These results suggested that there was crosstalk between phosphorylation and ubiquitination in the WNK4 protein, and high salt may downregulate its phosphorylation and, in turn, decrease its ubiquitination, leading to a decrease in WNK4 degradation. This eventually resulted in an increase in the abundance of WNK4 protein.
\end{abstract}

\section{Introduction}

The with no lysine (WNK; including WNK1, WNK2, WNK3 and WNK4) kinases are serine/threonine kinases lacking an ATP-bound lysine in the catalytic domain subtype II (1). WNK4 is highly expressed in the kidney, where it regulates diverse ion transporters and channels, including the Na-Cl co-transporter (NCC) (2-4). WNK4 appears to act as a molecular switch that can balance $\mathrm{NaCl}$

Correspondence to: Professor Yanyan Zhao, Department of Clinical Genetics, Shengjing Hospital of China Medical University, 36 San Hao Street, Shenyang, Liaoning 110003, P.R. China

E-mail: yyzhao@sj-hospital.org

*Contributed equally

Key words: high salt, WNK4, ubiquitination, phosphorylation, hypertension reabsorption and $\mathrm{K}^{+}$secretion, thereby maintaining blood pressure. A mutation in WNK4 (D561A) is responsible for pseudohypoaldosteronism type II (PHA II) (5), which is characterized by familial hyperkalemia and salt-sensitive hypertension $(6,7)$. Our previous study reported that polymorphism of WNK4 was associated with hypertension (8). Therefore, it has been demonstrated that WNK4 is involved in the pathophysiological processes of fluid and electrolyte perturbations and hypertension. Transcriptional regulation and post-translational modification contribute toward the regulation of protein abundance in tissues and cells. Our previous study described several mechanisms of WNK4 transcriptional regulation (9-11), but little is known regarding its post-translational modification.

Ubiquitination is one of the most important post-translational modifications. It has been proposed that WNK4 is a substrate-recognition protein of the Cullin 3-based E3 ligase complex, which targets WNK4 for ubiquitylation and degradation. It has also been reported that WNK4 protein may be bound by Kelch-like 3 (KLHL3) and Kelchlike 2 (KLHL2) $(12,13)$. Furthermore, impaired KLHL3 was suggested to mediate the ubiquitination of WNK4, thereby causing hypertension (14). This suggests that the ubiquitination and degradation of WNK4 serves important roles in its function. However, the exact site of ubiquitination in the WNK4 protein has remained unclear. In addition to ubiquitination, phosphorylation is also a normal post-translational modification used in eukaryotic cells to adjust and control the protein activity and function. Ubiquitination and phosphorylation are usually relatively independent $(13,15)$, but sometimes do affect each other. For example, it has been proposed that during hypoxia, phosphorylation is required for $\mathrm{Na}, \mathrm{K}-\mathrm{ATP}$ ase ubiquitination (16). However, it has remained unclear whether there is an interaction between ubiquitination and phosphorylation in WNK4.

It is well known that high salt levels are the primary cause of high blood pressure $(17,18)$; and the present study reported that mice fed a high-salt diet have increased blood pressure as well as increased sodium excretion (19). The purpose of the present study was to determine how high salt regulates WNK4 expression. The aim of the present study was to identify the site of ubiquitination in the WNK4 protein and to discover the mechanism by which high salt levels increase WNK4 protein through crosstalk between the ubiquitination and phosphorylation of WNK4. 


\section{Materials and methods}

Animals. Experiments were performed on 12-18-weekold C57BL/6 male mice (provided by the Department of Laboratory Animal Science of China Medical University) weighing 25-30 g. A total of 30 male mice were maintained and housed in stainless steel, wire-bottomed cages on a 12-h light/dark cycle at an ambient temperature of $23 \pm 2^{\circ} \mathrm{C}$ and $60 \%$ humidity. The mice were fed a diet containing either a normal salt level $(0.4 \% \mathrm{NaCl})$ or a high level of salt $(4 \% \mathrm{NaCl})$ for 2, 4, 6 and 8 weeks, and were then euthanized with $\mathrm{CO}_{2}$ at an air replacement rate of $20 \% / \mathrm{min}$ at the same age (20 weeks). Water and food were provided to all animals ad libitum. All animal experimental protocols were approved by the Ethics Committee of Shengjing Hospital of China Medical University.

Cell culture and stimulation. HEK293 cells were provided by the Department of Genetics, China Medical University and cultured in Dulbecco's modified Eagle's medium (DMEM; Gibco; Thermo Fisher Scientific, Inc.), supplemented with $10 \%$ fetal bovine serum (FBS; Gibco; Thermo Fisher Scientific, Inc.), $100 \mu \mathrm{g} / \mathrm{ml}$ penicillin and $100 \mu \mathrm{g} / \mathrm{ml}$ streptomycin (Gibco; Thermo Fisher Scientific, Inc.) at $37^{\circ} \mathrm{C}$ in a humidified atmosphere with $5 \% \mathrm{CO}_{2}$ in air. Subsequently, the cells were seeded onto a 6 -well plate and treated with $\mathrm{NaCl}$ (40 $\mathrm{mM}$ extra $\mathrm{NaCl}$ added to complete medium for $72 \mathrm{~h}$ ) or with mannitol ( $80 \mathrm{mM}$ for $72 \mathrm{~h}$ ) as a control. The medium was replaced every 2 days. Mycoplasma testing was performed on this cell line to ensure that the cells had not been contaminated by mycoplasma.

Construct of mutant plasmid vectors and transfections. Expression plasmids for HA-tagged human WNK4 were established as previously described (8). The following primers were used to construct the deleted plasmid vectors: K1010 forward, 5'-TGAAGAGGGACCGCAGCTTGTTGGGCGTTT-3' and reverse, 5'-CAAGCTGCGGTCCCTCTTCAGAGATGG GCG-3'; K1023 forward, 5'-GACTTCATCCGAACCG GCTGAGC CTCTTCC-3' and reverse, 5'-CAGCCGGT TCGGATGAAGTCACTTGGAAAC-3'; K1092 forward, 5'-AGATGATGGGGAACCCCAAGTTGGGGGCAG-3' and reverse, 5'-CTTGGGGTTCCCCATCATCTCCTTCCT CCTCA-3'; and S1022 forward, 5'-TTGGGCGTTTCCA AGTGACTTCAAAGGAAC-3' and reverse, 5'-GGCTCA GCCGGTTCCTTTGAAGTCACTTGG-3'

All constructs were verified by sequencing. HEK 293 cells $\left(3 \times 10^{5}\right.$ cells $/ 6-\mathrm{cm}$ dish) were transfected with the indicated amount of expression constructs using the jetPRIME ${ }^{\circledR}$ transfection reagent (Polyplus-transfection ${ }^{\circledR}$ ). Cell transfection was performed at $37^{\circ} \mathrm{C}$ for $6 \mathrm{~h}$, the transfection regent was removed and fresh DMEM medium was added. At $48 \mathrm{~h}$ following cell transfection, cells were used for subsequent experimentation.

Co-immunoprecipitation (Co-IP) and western blotting $(W B)$. The renal samples were immediately frozen at $-80^{\circ} \mathrm{C}$ after being removed from the mice. A total of $50 \mathrm{mg}$ kidney tissue was obtained and cut into pieces, followed by washing with $1 \mathrm{ml}$ PBS. Renal protein samples were prepared by homogenizing the frozen tissues in RIPA lysis buffer (cat. no. P0013B; Beyotime Biotechnology, Inc.) containing protease inhibitors (cat. no. 5871S; Cell Signaling Technology, Inc.). The cell proteins were prepared in lysis buffer containing protease and phosphatase inhibitors. The protein concentration was determined using the Bradford method with 5\% BSA as the washing reagent. For IP, 500 ug protein samples were pre-incubated with a primary antibody against ubiquitin (Ub; cat. no. 3933; dilution 1:1,000; Cell Signaling Technology, Inc.), HA-tag (cat. no. 26183; dilution 1:1,000; Thermo Fisher Scientific, Inc.) and Phospho-(Ser/Thr) (P-S/T; cat. no. 9631S; dilution 1:1,000; Cell Signaling Technology, Inc.) by rotating $(8 \mathrm{x} \mathrm{g})$ at $4^{\circ} \mathrm{C}$ overnight, followed by the addition of $20 \mu \mathrm{l}$ protein A/G PLUS-Agarose (cat. no. sc-2003; Santa Cruz Biotechnology, Inc.; ) and a further incubation with rotation $\left(8 \mathrm{x} \mathrm{g}, 37^{\circ} \mathrm{C}\right)$ for $1 \mathrm{~h}$. A total of $500 \mu \mathrm{g}$ of each protein sample in $20 \mu \mathrm{l}$ protein $\mathrm{A} / \mathrm{G}$ beads was collected and washed with lysis buffer three times. Immunoprecipitates were resolved by $8 \%$ SDS-polyacrylamide gel electrophoresis (PAGE) and analyzed by western blotting. For western blot analysis, $50 \mu \mathrm{g}$ protein samples were subjected to 8 or $10 \%$ SDS-PAGE and transferred onto polyvinylidene difluoride membranes (BioRad Laboratories, Inc.). The membranes were blocked with 5\% dry skimmed milk in TBS containing $0.1 \%$ Tween-20 (in phosphorylation experiments, the membranes were blocked with 5\% BSA buffer in TBS containing 0.1\% Tween-20) and incubated with primary antibodies against WNK4 (cat. no. 5713; dilution 1:1,000; Cell Signaling Technology, Inc.), Phospho-(Ser/Thr) (P-S/T; cat. no. 9631S; dilution 1:1,000; Cell Signaling Technology, Inc.), ubiquitin (Ub, cat. no. 3933; dilution 1:1,000; Cell Signaling Technology, Inc.), HA-tag (cat. no. 26183; dilution 1:1,000; Thermo Fisher Scientific, Inc.) and GAPDH (cat. no. 60004-1-Ig; dilution 1:10,000; Proteintech Group, Inc.), prior to being incubated with horseradish peroxidase-conjugated secondary antibodies, goat anti-rabbit IgG-HRP (cat. no. sc-2004; dilution 1:5,000; Santa Cruz Biotechnology, Inc.) and goat anti-mouse IgG-HRP (cat. no. sc-2005; dilution 1:5,000; Santa Cruz Biotechnology, Inc.) for $1 \mathrm{~h}$ at room temperature. The results were visualized using an enhanced chemiluminescence kit obtained from Thermo Fisher Scientific, Inc. Protein levels were normalized using ImageJ software (version 1.8.0; National Institutes of Health).

Forecasting. The UbPred database (www.ubpred.org) was used to identify the ubiquitination sites in the WNK4 protein. Potential phosphorylation sites of WNK4 were predicted with Netphos2.0 (www.cbs.dtu.dk/services/NetPhos/) and Musite software (Version 1.0; Digital Biology Laboratory, Inc.).

Statistical analysis. Data are expressed as the mean \pm standard error of the mean of three independent experiments. Bar graphs were constructed using GraphPad Prism 5 (Prism 5 for Windows; GraphPad Software, Inc.). Student's unpaired two-tailed test or one-way analysis of variance followed by Dunnett's test was used to assess the statistical significance of differences among different groups using the statistical software SPSS (version 17.0 for Windows; SPSS, Inc.). $\mathrm{P}<0.05$ was considered to indicate a statistically significant difference. 

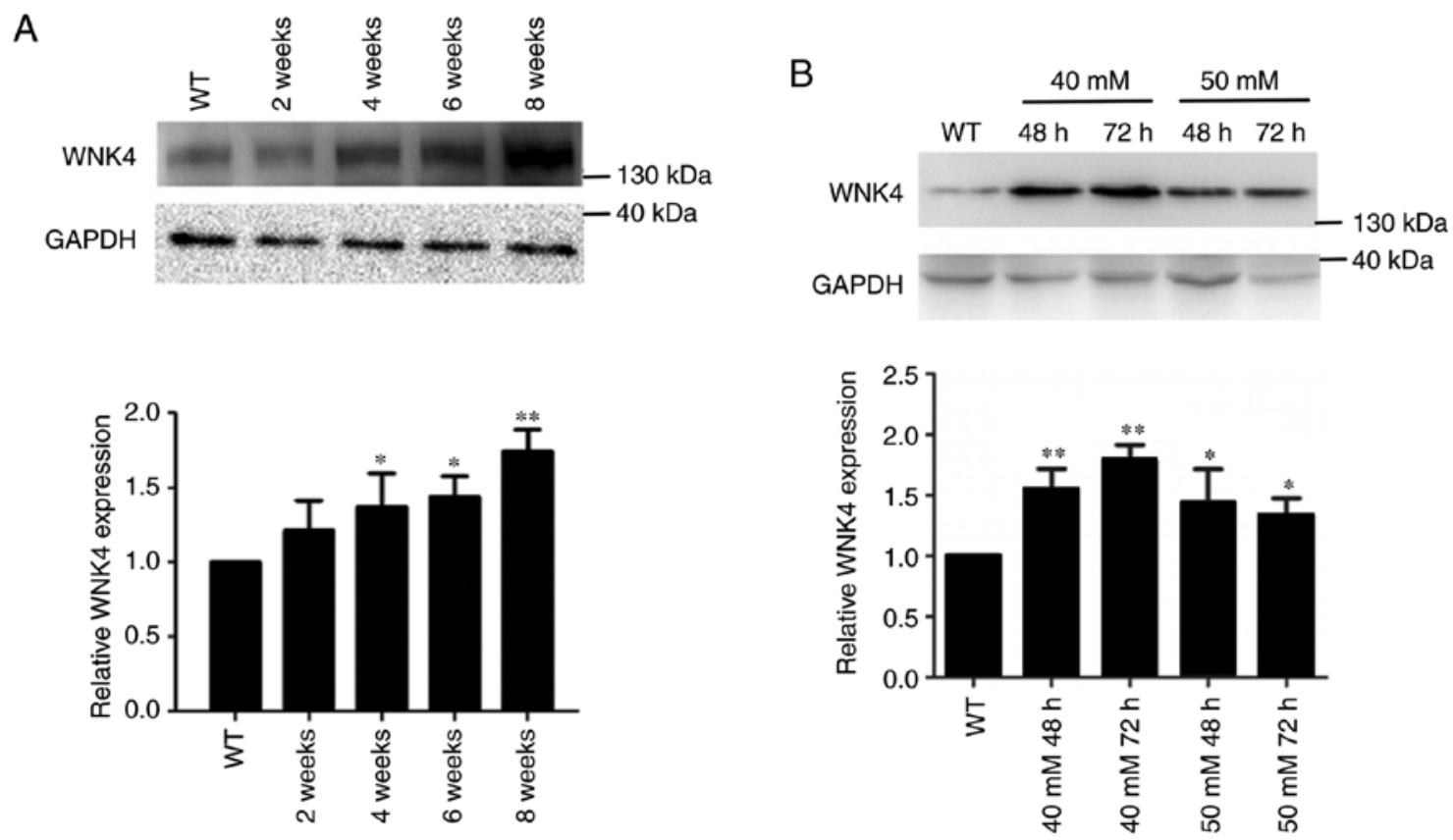

Figure 1. High salt stimulation increases WNK4 expression in mouse kidneys and HEK293 cells. (A) Mice were fed a high salt diet for different weeks. Western blot analysis was used to determine WNK4 protein expression in renal tissue. WT $(n=6)$, HS $(n=4)$. (B) Western blot analysis was used to analyze WNK4 protein expression in high-salt stimulated HEK293 cells. All experiments were repeated three times. ${ }^{*} \mathrm{P}<0.05 ;{ }^{* * *} \mathrm{P}<0.01$. WNK4, with no lysine 4 ; WT, wild-type.

\section{Results}

High salt increases the expression of WNK4. Mice were fed a high-salt diet for 2, 4, 6 or 8 weeks, after which the expression of the WNK4 protein in the kidney was determined by western blotting. The abundance of WNK4 in the kidneys of high-salt-fed mice was significantly higher than that in the kidney of wild-type (WT) mice (Fig. 1A). Since the level of WNK4 increased with increasing duration of high-salt intake, samples were selected after 8 weeks of feeding for the follow-up experiments. Similarly, as shown in Fig. 1B, WNK4 was upregulated in HEK293 cells following salt stimulation under different conditions (40 or $50 \mathrm{mM}$ for either 48 or $72 \mathrm{~h}$ ). As the increase in WNK4 protein was most pronounced at $40 \mathrm{mM}$ for $72 \mathrm{~h}$, these conditions were used in the subsequent experiments.

High-salt conditions reduces WNK4 ubiquitination. To determine the ubiquitination of WNK4 under high-salt conditions, Co-IP was used to detect the interactions between WNK4 and Ub. As shown in Fig. 2A, in high-salt-fed mice, the level of binding of Ub to WNK4 was significantly lower than that in WT mice. In addition, in HEK293 cells transfected with the HA-tagged WNK4 expression vector, the binding of Ub to WNK4 was lower under high-salt simulation than in the control cells (Fig. 2B). These findings suggested that the increased abundance of WNK4 induced by high salt was caused, at least in part, by a decrease in WNK4 ubiquitination and degradation.

Identification of the ubiquitination sites in the WNK4 protein. When attempting to determine the ubiquitination sites in the WNK4 protein, three lysine residues (K1010, K1023 and K1092) with high confidence scores were identified using
UbPred software, a sketch map of which is shown in Fig. 3A. Plasmids containing WNK4 with mutations in each lysine residue (K1010, K1023 and K1092) were constructed. WNK4 protein expression levels were greatly increased when the K1023 lysine was deleted, but only slightly or not increased when the K1092 or K1010 residue was deleted, compared with the level for WT WNK4 (Fig. 3B). Next, Co-IP was used to determine the ability of mutated $\mathrm{WNK} 4$ proteins to bind $\mathrm{Ub}$, and the three mutated forms of WNK4 all exhibited decreased binding to $\mathrm{Ub}$, compared with that of WT WNK4, particularly when the K1023 site was mutated (Fig. 3C). These results demonstrated that the K1023 site was the most important for ubiquitination of the WNK4 protein.

Phosphorylation of WNK4 is involved in its ubiquitination. It is known that crosstalk can occur between phosphorylation and ubiquitination under certain conditions (16); therefore, we hypothesized that phosphorylation of WNK4 may contribute toward its ubiquitination under high-salt conditions. WNK4 phosphorylation was detected by Co-IP, immunoprecipitated with anti-phosphorylated serine or threonine antibody $(\mathrm{P}-\mathrm{S} / \mathrm{T})$ and blotted with an anti-HA-tag antibody. As shown in Fig. 4A, in high-salt-fed mice, WNK4 phosphorylation was significantly decreased following high-salt stimulation, compared with the level in unstimulated kidney; in HEK293 cells transfected with HA-tagged WNK4 expression vector, the phosphorylation level of WNK4 was also lower under high-salt simulation than in unstimulated HEK293 cells. Potential phosphorylation sites of WNK4 were predicted using Netphos2.0 and Musite software. Next, the putative phosphorylation site, S1022, was selected to construct a site-mutated WNK4 protein, as this site is located proximal to the K1023 ubiquitination site. Using Co-IP, it was found that the binding of Ub to the WNK4-S1022 mutant was significantly lower than that to wild-type WNK4 
A
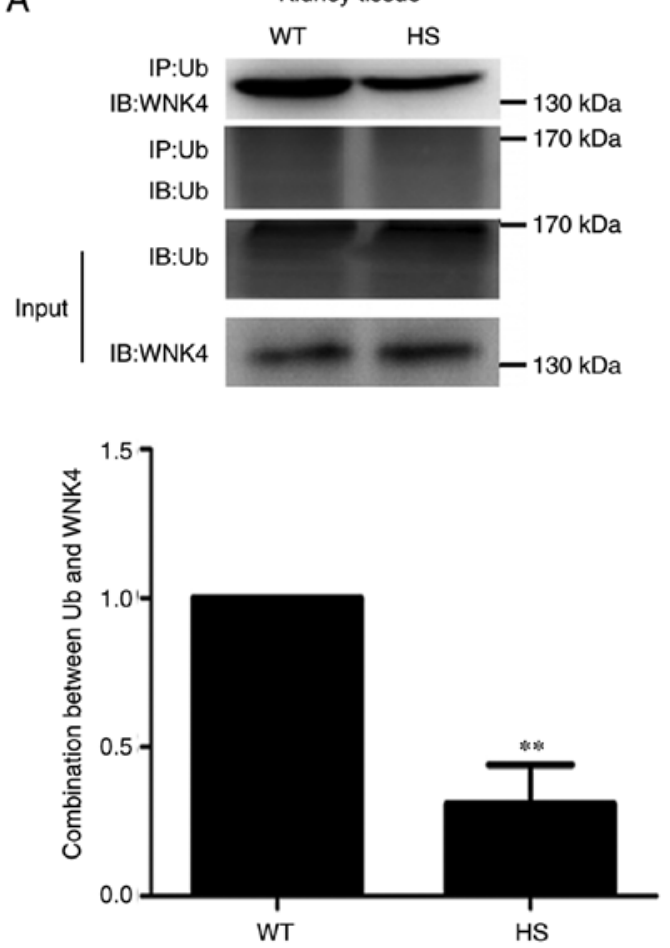

B
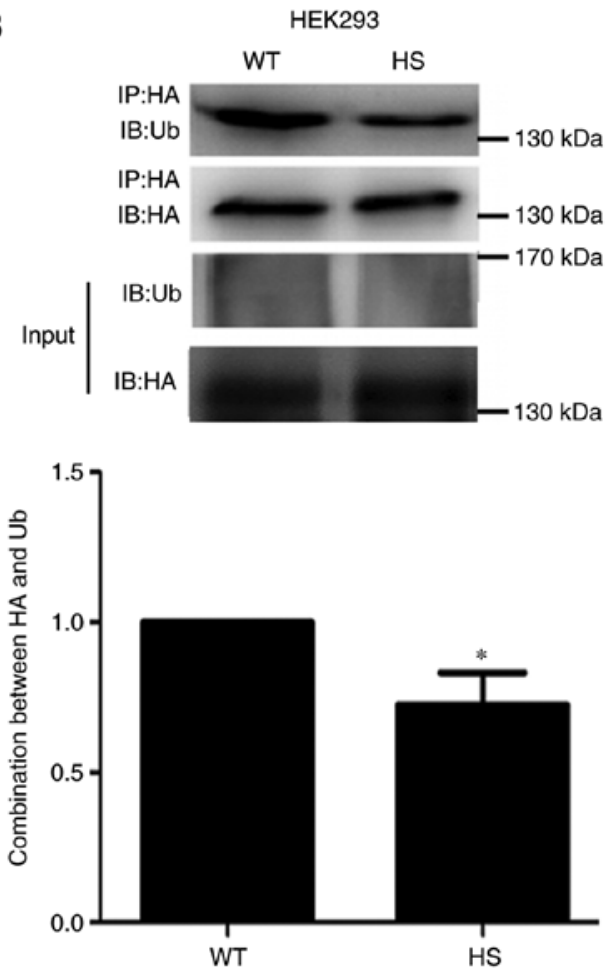

Figure 2. High-salt conditions decreased the ubiquitination of WNK4. (A) WNK4 binding to Ub was detected using Co-IP with an anti-Ub antibody followed by western blot with an anti-WNK4 antibody in mouse kidney. WT $(n=6)$, HS $(n=4)$. (B) WNK4 and Ub binding was analyzed using Co-IP with an anti-HA-tag antibody followed by western blot analysis with an anti-Ub antibody in HEK293 cells. All experiments were repeated three times. ${ }^{*} \mathrm{P}<0.05 ;{ }^{* *} \mathrm{P}<0.01$. WNK4, with no lysine 4; Ub, ubiquitin; Co-IP, co-immunoprecipitation; WT, wild-type; HS, high-salt; HA, human influenza hemagglutinin.

A

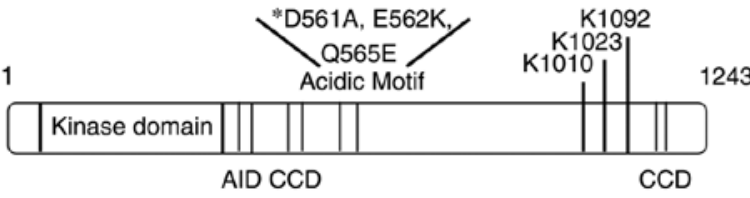

B
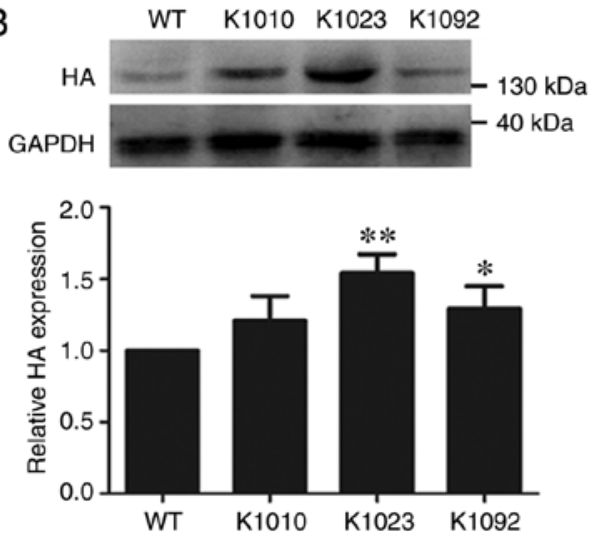

C
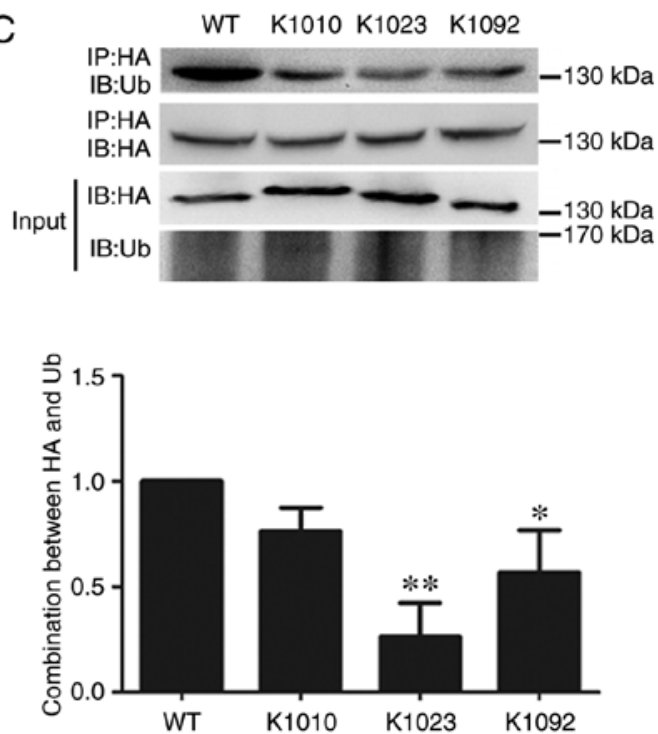

Figure 3. K1023 is the ubiquitination site of WNK4. (A) UbPred software was used to predict ubiquitination sites for WNK4. The diagram showed three high confidence predicted ubiquitination sites for WNK4. (B) Western blotting was used to analyze WNK4 protein expression after mutation of each of the three K residues. (C) The binding of WNK4 to Ub was detected after mutation of each of the three $\mathrm{K}$ residues. All trials were repeated three times. ${ }^{*} \mathrm{P}<0.05$; ${ }^{* *} \mathrm{P}<0.01$. WNK4, with no lysine 4; AID, auto-inhibitory domain; CCD, coiled-coil domain; Ub, ubiquitin; WT, wild-type; HA, human influenza hemagglutinin.

(Fig. 4B), implying that the phosphorylation of WNK4 at position $\mathrm{S} 1022$ affects the binding between WNK4 and Ub. These findings demonstrated that the decreased phosphorylation of WNK4 under high-salt conditions may result in its decreased ubiquitination and this process is shown in Fig. 4C.

\section{Discussion}

The present study identified that WNK4 protein expression was increased under high-salt conditions in mice and in vitro, which was consistent with a previous report (20). Furthermore, 
A
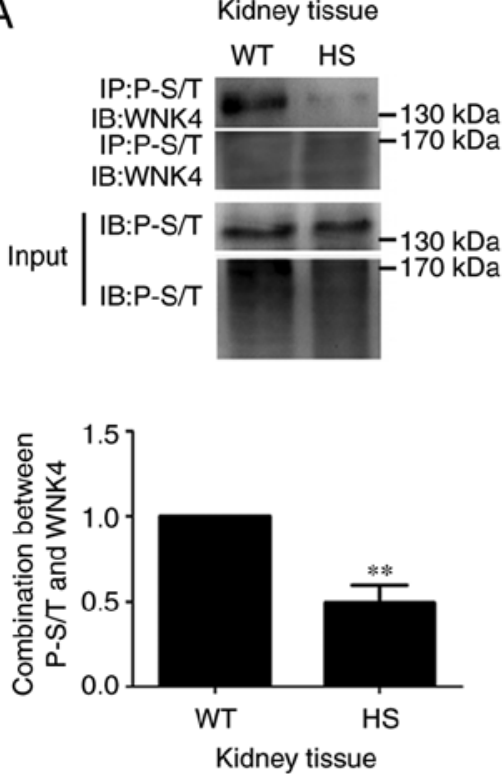

C

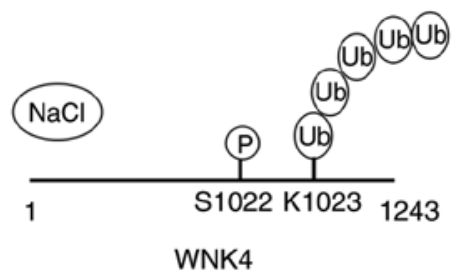

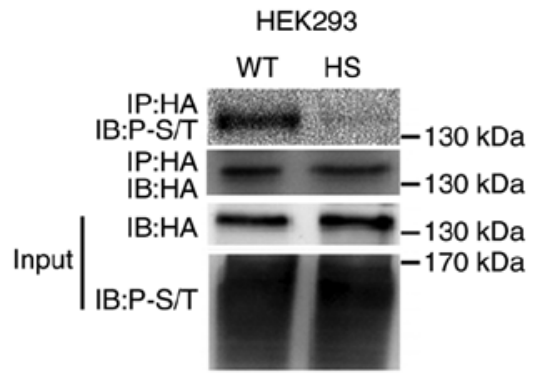
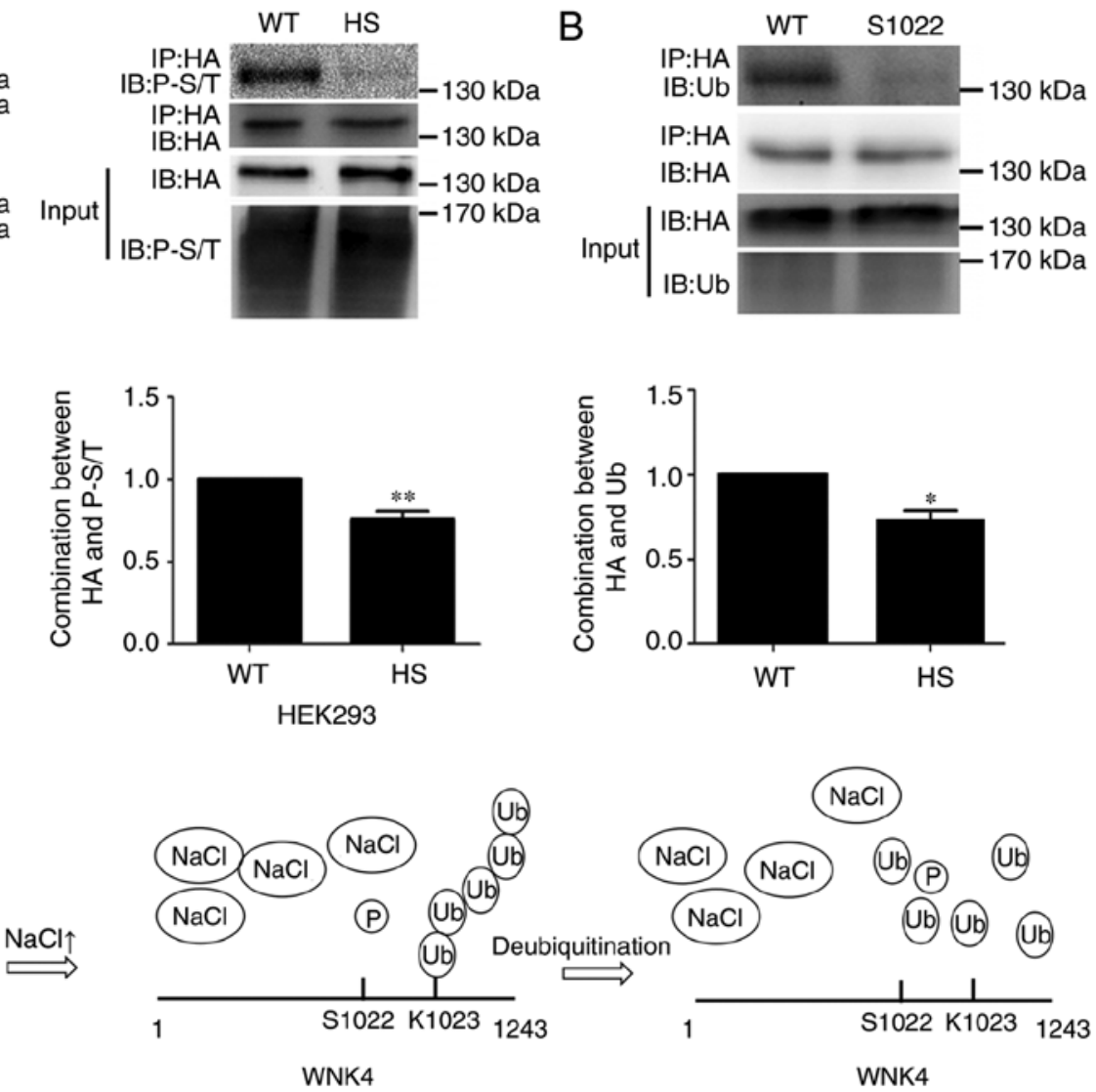

Figure 4. WNK4 protein exhibited crosstalk between its phosphorylation and ubiquitination sites. (A) The binding of WNK4 to P-S/T was detected by coimmunoprecipitation in mice or HEK293 cells. (B) The binding of WNK4 to Ub was detected after the S1022 site was deleted. All experiments were repeated three times. ${ }^{*} \mathrm{P}<0.05 ;{ }^{* *} \mathrm{P}<0.01$. (C) The pattern diagram of the potential mechanism. When the salt concentration increases, the phosphorylation of WNK4 protein decreases, which in turn decreases its ubiquitination. WNK4, with no lysine 4; P-S/T, phosphorylated serine or threonine; Ub, ubiquitin; WT, wild-type; HS, high-salt; HA, human influenza hemagglutinin.

it was demonstrated that high salt downregulated phosphorylation at the $\mathrm{S} 1022$ site, which in turn decreased the ubiquitination of WNK4 protein, leading to a decrease in its degradation and thus an increase in its abundance.

The WNK4 protein possesses a kinase domain at its $\mathrm{N}$-terminus, which is abutted by an auto-inhibitory domain and then two putative coiled-coil domains (21). After the first putative coiled-coil domain, there is a region called the 'acidic domain', which binds to an E3 ligase complex, including Cullin3-KLHL3 and Cullin3-KLHL2 $(22,23)$. Next, WNK4 protein connects to ubiquitin molecules near specific lysine residues. Finally, the ubiquitinated WNK4 protein is degraded by proteasomes. Numerous studies have reported that mutant KLHL3 and Cullin3 molecules may decrease the ubiquitination of WNK4 $(24,25)$. In addition, WNK4 mutations (E562K, Q565E and D561A) $(5,13,26)$ have been reported to inhibit the binding of WNK4 to KLHL3; however, the exact site of ubiquitination in the WNK4 protein remained unclear. In the present study, UbPred software was used to predict three ubiquitination sites with high confidence (K1010, K1023 and K1092) and constructed the respective mutant WNK4 expression plasmids. These experiments demonstrated that deletion of the K1023 site resulted in increased protein levels of WNK4 associated with decreased ubiquitin binding, compared with that for wild-type WNK4. Therefore, the present study was the first to identify an important ubiquitination site for the WNK4 protein at K1023.

In addition to ubiquitination, phosphorylation is another important post-translational modification affecting protein activity and function. Protein phosphorylation may occur at multiple amino acids, but mostly at serine or threonine (27), and it has been reported that high salt may decrease p38 and SPAK phosphorylation $(28,29)$. Recent evidence has suggested that there are three primary ways in which phosphorylation regulates the ubiquitination of proteins. To begin with, phosphorylation may positively and negatively regulate the activity of the E3 ligase responsible for Ub transfer. Additionally, phosphorylation promotes the identification of E3 ligase by producing a phosphodegron. Furthermore, phosphorylation may influence ubiquitination by regulating the substrate/ligase interaction at the level of subcellular compartmentalization $(30,31)$. The present study reported that the phosphorylation of WNK4 was significantly decreased in HEK293 cells following high-salt stimulation, compared with that in unstimulated HEK293 cells, which is similar to reports suggesting that high salt decreases SPAK phosphorylation (29). We hypothesized that the phosphorylation of WNK4 contributes toward the process of WNK4 ubiquitination during high-salt stimulation. In certain proteins, the ubiqui- 
tination and phosphorylation sites are thought to be adjacent to each other $(16,31,32)$. Therefore, using the phosphorylation sites predicted by Musite and Netphos 2.0 software, the phosphorylation site that was nearest to the K1023 ubiquitination site was selected to be mutated. It was observed that WNK4S1022 exhibited decreased binding to Ub, suggesting that phosphorylation of $\mathrm{S} 1022$ was required for the ubiquitination of WNK4. Although the results of the present study demonstrated that high-salt conditions may decrease phosphorylation of the WNK4 protein, it is not possible to determine whether this was due to a decreased phosphorylation or increased dephosphorylation. Further studies are required to investigate whether phosphorylation or dephosphorylation involved in this process.

In conclusion, the results of the present study demonstrated that high-salt conditions would result in increased expression of WNK4 protein. The mechanism behind this may involve high salt inhibiting the ubiquitination of the WNK4 protein by decreasing its phosphorylation, which then decreases the degradation of WNK4 and eventually results in an increases level of it. These findings suggested that salt-sensitive hypertension may be successfully addressed through the targeted treatment of WNK4 protein ubiquitination or pharmacological inhibition of the WNK4 protein.

\section{Acknowledgements}

Not applicable.

\section{Funding}

The present study was supported by the National Key Special Projects of China (grant nos. 2016YFC1000700 and 2016YFC1000702), the 345 Talent Project (grant no. M0298) and the Free Researcher Fund (grant no. ME57) of Shengjing Hospital.

\section{Availability of data and materials}

The datasets used and/or analyzed during the current study are available from the corresponding author on reasonable request.

\section{Authors' contributions}

$\mathrm{XZ}, \mathrm{GL}$ and $\mathrm{YZ}$ designed the research and revised the manuscript. XZ, GL, YZ and JT performed the experiments and drafted the manuscript. JT and SL performed data analysis. All authors read and approved the final manuscript.

\section{Ethics approval and consent to participate}

All experimental protocols were approved by the Institutional Animal Care and Use Committee of Shengjing Hospital and were conducted in strict accordance with the National Institutes of Health guidelines for the use of experimental animals.

\section{Patient consent for publication}

Not applicable.

\section{Competing interests}

The authors declare that they have no competing interests.

\section{References}

1. Shekarabi M, Zhang J, Khanna AR, Ellison DH, Delpire E and Kahle KT: WNK kinase signaling in ion homeostasis and human disease. Cell Metab 25: 285-299, 2017.

2. Subramanya AR, Yang CL, McCormick JA and Ellison DH: WNK kinases regulate sodium chloride and potassium transport by the aldosterone-sensitive distal nephron. Kidney Int 70 : 630-634, 2006.

3. Tang BL: (WNK)ing at death: With-no-lysine (Wnk) kinases in neuropathies and neuronal survival. Brain Res Bull 125: 92-98, 2016.

4. Hadchouel J, Ellison DH and Gamba G: Regulation of renal rlectrolyte transport by WNK and SPAK-OSR1 kinases. Annu Rev Physiol 78: 367-389, 2016.

5. Chu PY, Cheng CJ, Wu YC, Fang YW, Chau T, Uchida S, Sasaki S, Yang SS and Lin SH: SPAK deficiency corrects pseudohypoaldosteronism II caused by WNK4 mutation. PLoS One 8: e72969, 2013.

6. Gordon RD: Syndrome of hypertension and hyperkalemia with normal glomerular filtration rate. Hypertension 8: 93-102, 1986.

7. O'Shaughnessy KM: Gordon Syndrome: A continuing story. Pediatr Nephrol 30: 1903-1908, 2015.

8. Sun Z, Li Y, Lu J, Ding Q, Liang Y, Shi J, Li-Ling J and Zhao Y: Association of Ala589Ser polymorphism of WNK4 gene with essential hypertension in a high-risk Chinese population. J Physiol Sci 59: 81-86, 2009.

9. Li C, Li Y, Li Y, Liu H, Sun Z, Lu J and Zhao Y: Glucocorticoid repression of human with-no-lysine $(\mathrm{K})$ kinase-4 gene expression is mediated by the negative response elements in the promoter. Mol Endocrinol 40: 3-12, 2008.

10. Li M, Zhao Y, Li Y, Li C, Chen F, Mao J and Zhang Y: Upregulation of human with-no-lysine kinase-4 gene expression by GATA-1 acetylation. Int J Biochem Cell Biol 41: 872-878, 2009.

11. Zhang Y, Li C, Li W and Zhao Y: Estrogen regulation of human with-no-lysine $(\mathrm{K})$ kinase-4 gene expression involves AP-1 transcription factor. Mol Cell Endocrinol 332: 140-148, 2011.

12. Schumacher FR, Sorrell FJ, Alessi DR, Bullock AN and Kurz T: Structural and biochemical characterization of the KLHL3WNK kinase interaction important in blood pressure regulation. Biochem J 460: 237-246, 2014.

13. Takahashi D, Mori T, Wakabayashi M, Mori Y, Susa K, Zeniya M, Sohara E, Rai T, Sasaki S and Uchida S: KLHL2 Interacts with and ubiquitinates WNK kinases. Biochem Biophys Res Commun 437: 457-462, 2013.

14. Susa K, Sohara E, Rai T, Zeniya M, Mori Y, Mori T, Chiga M, Nomura N, Nishida H, Takahashi D, et al: Impaired degradation of WNK1 and WNK4 kinases causes PHAII in mutant KLHL3 knock-in mice. Hum Mol Genet 23: 5052-5060, 2014.

15. Ardito F, Giuliani M, Perrone D, Troiano G and Lo Muzio L: The crucial role of protein phosphorylation in cell signaling and its use as targeted therapy (Review). Int J Mol Med 40: 271-280, 2017.

16. Dada LA, Welch LC, Zhou G, Ben-Saadon R, Ciechanover A and Sznajder JI: Phosphorylation and ubiquitination are necessary for Na,K-ATPase endocytosis during hypoxia. Cell Signal 19: 1893-1898, 2007.

17. Pilic L, Pedlar CR and Mavrommatis Y: Salt-sensitive hypertension: Mechanisms and effects of dietary and other lifestyle factors. Nutr Rev 74: 645-658, 2016.

18. Friso S, Carvajal CA, Fardella CE and Olivieri O: Epigenetics and arterial hypertension: The challenge of emerging evidence. Transl Res 165: 154-165, 2015.

19. Wu J, Liu X, Lai G, Yang X, Wang L and Zhao Y: Synergistical effect of 20-HETE and high salt on NKCC2 protein and blood pressure via ubiquitin-proteasome pathway. Hum Genet 132: 179-187, 2013.

20. Lai L, Feng X, Liu D, Chen J, Zhang Y, Niu B, Gu Y and Cai H: Dietary salt modulates the sodium chloride cotransporter expression likely through an aldosterone-mediated WNK4ERK1/2 signaling pathway. Pflugers Arch 463: 477-485, 2012. 
21. Wang Z, Subramanya AR, Satlin LM, Pastor-Soler NM, Carattino MD and Kleyman TR: Regulation of large-conductance $\mathrm{Ca}_{2}^{+}$-activated $\mathrm{K}^{+}$channels by WNK4 kinase. Am J Physiol Cell Physiol 305: C846-C853, 2013.

22. Kliuk-Ben Bassat O, Carmon V, Hanukoglu A, Ganon L, Massalha E, Holtzman EJ, Farfel Z and Mayan H: Familial hyperkalemia and hypertension (FHHt) and KLHL3: Description of a family with a new recessive mutation (S553L) compared to a family with a dominant mutation, Q309R, with analysis of urinary sodium chloride cotransporter. Nephron 137: 77-84, 2017.

23. Kasagi Y, Takahashi D, Aida T, Nishida H, Nomura N, Zeniya M, Mori T, Sasaki E, Ando F, Rai T, et al: Impaired degradation of medullary WNK4 in the kidneys of KLHL2 knockout mice. Biochem Biophys Res Commun 487: 368-374, 2017.

24. Susa K, Sohara E, Takahashi D, Okado T, Rai T and Uchida S: WNK4 is indispensable for the pathogenesis of pseudohypoaldosteronism type II caused by mutant KLHL3. Biochem Biophys Res Commun 491: 727-732, 2017.

25. Ibeawuchi SR, Agbor LN, Quelle FW and Sigmund CD: Hypertension- causing mutations in Cullin3 protein impair RhoA protein ubiquitination and augment the association with substrate adaptors. J Biol Chem 290: 19208-19217, 2015.
26. Ohta A, Schumacher FR, Mehellou Y, Johnson C, Knebel A, Macartney TJ, Wood NT, Alessi DR and Kurz T: The CUL3KLHL3 E3 ligase complex mutated in Gordon's hypertension syndrome interacts with and ubiquitylates WNK isoforms: Disease-causing mutations in KLHL3 and WNK4 disrupt interaction. Biochem J 451: 111-122, 2013.

27. Lee HK: Synaptic plasticity and phosphorylation. Pharmacol Ther 112: 810-832, 2006

28. Dinarello CA: Hyperosmolar sodium chloride, p38 mitogen activated protein and cytokine-mediated inflammation. Semin Dial 22: 256-259, 2009.

29. Zeniya M, Sohara E, Kita S, Iwamoto T, Susa K, Mori T, Oi K, Chiga M, Takahashi D, Yang SS, et al: Dietary salt intake regulates WNK3-SPAK-NKCC1 phosphorylation cascade in mouse aorta through angiotensin II. Hypertension 62: 872-878, 2013.

30. Hunter T: The age of crosstalk: Phosphorylation, ubiquitination, and beyond. Mol Cell 28: 730-738, 2007.

31. Filipčík P, Curry JR and Mace PD: When worlds collidemechanisms at the interface between phosphorylation and ubiquitination. J Mol Biol 429: 1097-1113, 2017.

32. Kelm KB, Huyer G, Huang JC and Michaelis S: The internalization of yeast Ste6p follows an ordered series of events involving phosphorylation, ubiquitination, recognition and endocytosis. Traffic 5: 165-180, 2004. 